\title{
The Benefits of Patients' Information regarding Dietary Habits before Percutaneous Coronary Intervention: A 3- and 6-Month Follow-Up
}

\author{
Argyriou George ${ }^{1 *}$, Vasilopoulos George², Mavrogianni Georgia ${ }^{3}$, Toulia Georgia², \\ Louka Aikaterini' ${ }^{4}$, Louka Sofia ${ }^{4}$, Karagiannis Anastasios ${ }^{5}$, Lazos Gabriel ${ }^{1}$, \\ Marvaki Aikaterini' ${ }^{6}$, Kadda 0lga ${ }^{5}$
}

\author{
${ }^{1}$ Intensive Care Unit, 1st Department of Respiratory Medicine, National and Kapodistrian University of Athens, "Sotiria" General \\ Chest Diseases Hospital, Greece \\ ${ }^{2}$ Nursing Department, Technological Educational Institute of Athens, Greece \\ ${ }^{3}$ Cardiological Intensive Care Unit, “Konstantopoulio” General Hospital of N. Ionias, Greece \\ ${ }^{4}$ Graduates of Nursing Department, Technological Educational Institute of Athens, Greece \\ ${ }^{5}$ Cardiological Intensive Care Unit, Onassis Cardiac Surgery Centre, Greece \\ ${ }^{6}$ General Hospital of Katerini, Greece \\ Email: *ageorge2000@yahoo.com
}

How to cite this paper: George, A. George, V., Georgia, M., Georgia, T., Aikaterini, L., Sofia, L., Anastasios, K., Gabriel, L., Aikaterini, M. and Olga, K. (2018) The Benefits of Patients' Information regarding Dietary Habits before Percutaneous Coronary Intervention: A 3- and 6-Month Follow-Up. World Journal of Cardiovascular Diseases, 8, 307-318. https://doi.org/10.4236/wjcd.2018.86030

Received: April 19, 2018

Accepted: June 22, 2018

Published: June 25, 2018

Copyright $\odot 2018$ by authors and Scientific Research Publishing Inc. This work is licensed under the Creative Commons Attribution International License (CC BY 4.0).

http://creativecommons.org/licenses/by/4.0/ (c) (i) Open Access

\begin{abstract}
Aim: To evaluate the benefits of counseling intervention related to dietary habits changes on patients following percutaneous coronary intervention (PCI). Materials and Methods: A randomized counseling intervention study, with a 3- and 6-month follow-up was performed on 230 patients who underwent PCI. They were randomly allocated to the intervention $(n=93)$ or the control group $(\mathrm{n}=137)$. A 3- and 6-month telephone follow-up was performed for dietary habits evaluation. Data analysis was performed by using the statistical package SPSS, ver. 20. Results: Compared with control group, intervention group had higher prevalence of hypertension, history of diabetes and dyslipidemias and history of acute myocardial infraction, with no statistical difference. Moreover, patients in control group were more likely to be ex-smokers $(p=0.01)$. Post hoc tests using the Bonferroni correction revealed that mean TCHOL concentration while patients admitted to hospital differed statistically significantly between the time points of 3 and 6 months $(209 \pm 67 \mathrm{mg} / \mathrm{dl}$ vs $174 \pm 34 \mathrm{mg} / \mathrm{dl}$ vs $176 \pm 36 \mathrm{mg} / \mathrm{dl}), p=0.005$ and $p=0.042$ respectively. However, there was no statistical significant difference between 3 months and 6 months measurements. Mean glucose concentration while patients admitted to hospital differed statistically significantly between the time points of 3 and 6 months (108 $\pm 40 \mathrm{mg} / \mathrm{dl}$ vs $95 \pm 21 \mathrm{mg} / \mathrm{dl}$ vs $95 \pm 23 \mathrm{mg} / \mathrm{dl}), p=0.009$ and $p$ $=0.012$ respectively. However, there was no significant statistical difference
\end{abstract}


between 3 months and 6 months measurements, $(p=1.000)$. Conclusion: A nurse-led program regarding dietary habits modifications on patients undergoing PCI should be performed along with a long-term follow up after hospital discharge.

\section{Keywords}

Dietary Habits Intervention, MedDietScore, Percutaneous Coronary Intervention, Patient's Information

\section{Introduction}

Coronary heart disease (CHD) remains one of the leading causes of mortality worldwide; in 2012 an estimated 17.5 million people died from cardiovascular disease representing $31 \%$ of all global deaths [1]. Researchers [2] [3] continue to study and draw their attention to the risk factors responsible for CHD events. Risk factors are strongly associated with each other and often build on one another; such as excess body weight leads to diabetes of high blood pressure. Dietary interventions are proposed to initiate preferably during childhood so as to prevent coronary events in the early age [4].

The traditional Mediterranean diet has been described to moderate the development of CHD and reduce the risk of CHD events [5] [6]. This kind of diet is low in saturated fat and has 9 components: a high consumption of olive oil, legumes, cereals, fruits, and vegetables; a moderate to high consumption of fish, low to moderate consumption of dairy products (cheese, yogurt), moderate consumption of red wine and low consumption of meat [6].

This pattern can reduce the risk of developing an acute coronary syndrome (ACS) in people at high cardiovascular risk (primary prevention) [7] or those with established CHD (secondary prevention) [8]. The beneficial effects of dietary modifications are widely discussed in recent studies [9] [10] giving emphasis to cholesterol levels, weight and diabetes management, fiber and fish oils consumption.

Health care professionals have the opportunity while patients hospitalized to advice on risk factors and dietary habits modification [11]. The results of an education programme on patient outcome depend upon patients' adherence to recommendations. A well structured prevention programme can be developed with the support of a well organized health care system. Nurses led programmes are proved to be an effective way to reduce CVD events of high risk persons [12] and skilled nurses can provide education related to dietary habits so as to improve CVD's outcomes [13].

\section{Aim}

The aim of the present study was to evaluate the benefits of counseling interven- 
tion, related to dietary habits changes for patients following percutaneous coronary intervention (PCI).

\section{Materials and Methods}

\subsection{Study Design}

A randomized controlled single-blind counseling intervention study; random numbers tables were used, with 3 and 6 month follow-up.

\subsection{Participants}

During January 2011 to December 2012, 239 patients who underwent percutaneous coronary intervention were initially considered as appropriate to enroll in the study. Of them, 93 (intervention group) were randomly allocated lifestyle counseling while the remaining 137 patients (control group) received the regular instructions. According to the exclusion criteria 9 patient were finally excluded because: $\mathrm{n}=2$ were not able to cooperate and provide written informed consent; $\mathrm{n}=3$ were under specific dietary recommendations; $n=1$ was unable to understand the Greek language; $n=3$ participated in another trial at the same period of time, (Figure 1). Patient who had percutaneous coronary intervention for the first time were included to the study. The exclusion criteria for the participants were foreign language and absence of patients' informed consent. Moreover,

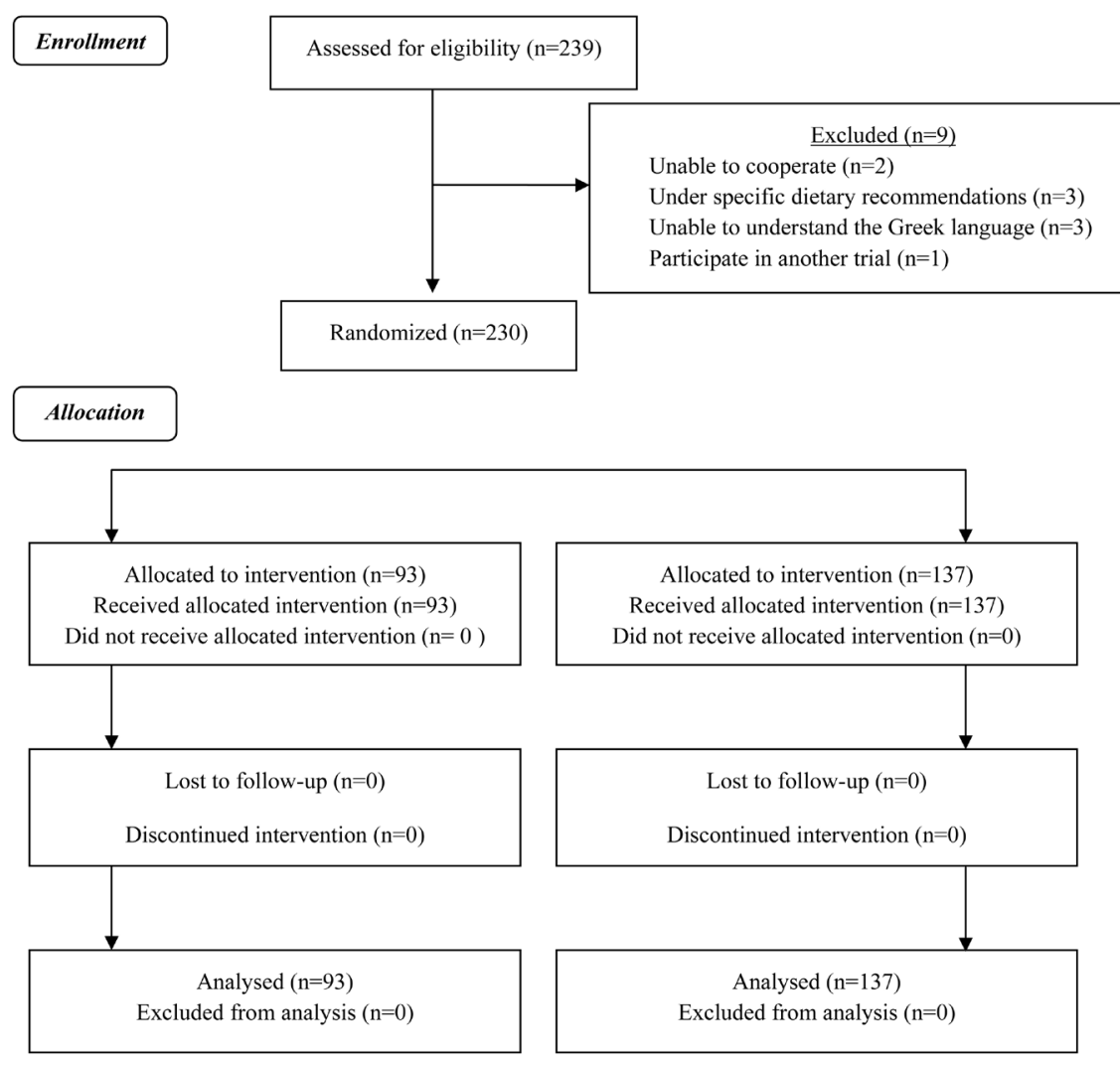

Figure 1. Flow chart of the randomized clinical trial. 
patients who participated in another trial during the same period of time were excluded.

\subsection{Procedure of the Intervention}

During the first interview with the patient, a questionnaire was completed by a specialized clinical nurse. The control group received regular oral instructions the usual recommendations from the nursing staff (according to the hospitals protocol). The hospitals protocol refers to simplified general instructions related to healthy diet. The intervention group received written information by administrating a booklet with specific dietary recommendations. The pattern of Mediterranean diet [14] was underlined. Specifically, they were consulted to consume non refined products, fruits, vegetables, olive-oil, in a daily basis; fish, poultry, and nuts in a weekly basis, and rarely sweets. Red meat and meat products should be in their monthly options; 4 servings per month). Moreover, they were instructed to retain a normal body weight or to consult a dietitian if necessary (i.e. patients with excess body weight). In order to follow patients' adherence to the Mediterranean dietary (MD) pattern, the MedDietScore (range 0 - 55) was used [14]. A higher value of MedDietScore indicates greater adherence to Mediterranean diet.

\subsection{Follow-Up}

Three and six months after hospital discharge a telephone follow up took place so as to assess patients' adherence to dietary advice. The main reason for choosing the 3 and 6 months time after hospital discharge was the fact that, according to literature, patients undergone stent implemention should be followed regularly (every three to six months) as this a high risk period for patients with acute coronary syndrome [15].

\subsection{Measurements}

Patients were interviewed by a specialized clinical nurse as for their demographic characteristics (age, sex, family status, financial status, educational status and profession) and their medical history (history and management of hypertension, diabetes, dyslipidaimias; family medical history of CVD and their current medication treatment). The Body Mass Index was also recorded.

\section{Ethical Considerations}

The necessary permission was obtained from each hospital's Ethics Committee. Patients were informed about the purpose of the study, the voluntary nature and the confidentiality of the research and a written informed consent was completed.

\section{Statistical Analysis}

Continuous variables are presented as mean values \pm standard deviation, and categorical variables as frequencies. Baseline characteristics were compared by 
applying chi-square test for categorical variables and independent samples t-test for continuous variables. A repeated measures ANOVA analysis so as One-way ANOVA analysis was applied. Data analysis was performed by using the statistical package SPSS, ver. 20.

\section{Results}

The demographic and baseline clinical characteristics of the studied sample are presented in Table 1. Compared with control group, intervention group had higher prevalence of hypertension, history of diabetes and dyslipidemias and history of acute myocardial infraction, with no statistical difference. Moreover, patients in control group were more likely to be ex-smokers $(p=0.01)$.

In Table 2, the results according to patient's outcome, adherence to MedDietScore and laboratory findings at 3 months following PCI are presented. As for laboratory findings, compared with control group, intervention group had lower total cholesterol and LDL concentration at 3 months following PCI but lower MedDietScore.

Table 1. Demographic and clinical characteristics of the studied sample who underwent percutaneous coronary intervention, $(\mathrm{N}=230)$.

\begin{tabular}{|c|c|c|c|}
\hline & $\begin{array}{l}\text { Intervention group } \\
\qquad(\mathrm{n}=93)\end{array}$ & $\begin{array}{l}\text { Control Group } \\
\quad(n=137)\end{array}$ & $p$-value \\
\hline \multicolumn{4}{|c|}{ Demographic characteristics } \\
\hline Gender (men), n (\%) & $72(39.8)$ & $109(60.2)$ & 0.40 \\
\hline \multicolumn{4}{|c|}{ Marital status } \\
\hline Married, n (\%) & $69(74.2)$ & $108(79.4)$ & \\
\hline Unmarried, n (\%) & $4(4.3)$ & $7(5.1)$ & \\
\hline Divorced, n (\%) & $6(6.5)$ & $5(3.7)$ & 0.65 \\
\hline Widowed, n (\%) & $14(15.1)$ & $16(11.8)$ & \\
\hline \multicolumn{4}{|c|}{ Financial status (annual income) } \\
\hline Bad (<9000 euro), n (\%) & $18(19.4)$ & $36(26.5)$ & \\
\hline Moderate (9001-18000 euro), n (\%) & $50(53.8)$ & $63(46.3)$ & \\
\hline Good (18001-28000 euro), n (\%) & $16(17.2)$ & $18(13.2)$ & 0.40 \\
\hline Very good (>28000 euro), n (\%) & $1(1.1)$ & $5(3.7)$ & \\
\hline No answer, n (\%) & $8(8.6)$ & $14(10.3)$ & \\
\hline \multicolumn{4}{|c|}{ Clinical characteristics } \\
\hline History of hypertension (Yes, n (\%)) & $39(41.9)$ & $70(51.1)$ & 0.10 \\
\hline History of diabetes (Yes, n (\%)) & $32(34.4)$ & $41(31.1)$ & 0.35 \\
\hline Family history of CVD (Yes, n (\%)) & $39(41.9)$ & $70(52.6)$ & 0.07 \\
\hline History of dyslipidemias (Yes, n (\%)) & $47(50.5)$ & $83(63.8)$ & 0.03 \\
\hline Smoker (at baseline) (Yes, n (\%)) & $23(26.1)$ & $35(28)$ & 0.44 \\
\hline Ex smoker (Yes, n (\%)) & $76(86.4)$ & $91(72.8)$ & 0.01 \\
\hline
\end{tabular}




\section{Continued}

\begin{tabular}{|c|c|c|c|}
\hline History of AMI (Yes, n (\%)) & $75(80.6)$ & $104(75.9)$ & 0.24 \\
\hline History of chronic kidney disease (Yes, n (\%)) & $14(15.1)$ & $16(11.7)$ & 0.29 \\
\hline \multicolumn{4}{|c|}{ Respiratory system } \\
\hline Normal (Yes, n (\%)) & $76(82.6)$ & $115(83.9)$ & \\
\hline COPD (Yes, n (\%)) & $15(16.3)$ & $21(15.3)$ & 0.93 \\
\hline Asthma (Yes, n (\%)) & $1(1.1)$ & $1(0.7)$ & \\
\hline Age (years) ${ }^{\mathrm{a}}$ & $60.8 \pm 11.5$ & $62.3 \pm 10.8$ & \\
\hline BMI $\left(\mathrm{Kg} / \mathrm{m}^{2}\right)(\text { baseline })^{\mathrm{a}}$ & $27.3 \pm 3.9$ & $27.6 \pm 4.4$ & 0.57 \\
\hline Total cholesterol (mg/dl) (baseline) ${ }^{\mathrm{a}}$ & $215.4 \pm 67.4$ & $196.7 \pm 56.3$ & 0.07 \\
\hline LDL cholesterol (mg/dl) (baseline) ${ }^{\mathrm{a}}$ & $126 \pm 34.5$ & $127.1 \pm 40.1$ & 0.86 \\
\hline HDL cholesterol (mg/dl) (baseline) ${ }^{\mathrm{a}}$ & $42.3 \pm 20.4$ & $43.8 \pm 17.0$ & 0.63 \\
\hline TGL (mg/dl) (baseline) $)^{\mathrm{a}}$ & $167 \pm 51$ & $161 \pm 75$ & 0.52 \\
\hline Blood glucose (mg/dl) (baseline) ${ }^{\mathrm{a}}$ & $156.9 \pm 100$ & $147 \pm 82.3$ & 0.45 \\
\hline
\end{tabular}

AMI: Acute Myocardial Infarction; COPD: Chronic Obstructive Pulmonary Disease; BMI: Body Mass Index; LDL: Low Density Lipoprotein; HDL: High Density Lipoprotein; TGL: Triglyceride; CVD: Cardiovascular Disease. ${ }^{\mathrm{a}}$ Mean \pm standard deviation.

Table 2. Results according to patient's outcome, adherence to MedDietScore and laboratory findings at 3 months following percutaneous coronary intervention.

\begin{tabular}{cccc}
\hline & $\begin{array}{c}\text { Intervention group } \\
(\mathrm{n}=93)\end{array}$ & $\begin{array}{c}\text { Control Group } \\
(\mathrm{n}=137)\end{array}$ & p-value \\
\hline Hospital resubmission, (Yes, n (\%)) & $18(20.5)$ & $28(22.4)$ & \\
3-months fatal outcome, (Yes, n (\%)) & 0 & $5(3.8)$ & \\
BMI (Kg/m $)^{\mathrm{a}}$ & $26.9 \pm 3.9$ & $27.3 \pm 4.2$ & 0.45 \\
MedDietScore $\left(\mathrm{range} 0\right.$ - 55) ${ }^{\mathrm{a}}$ & $29.1 \pm 4.7$ & $31.1 \pm 4.5$ & 0.003 \\
Total cholesterol (mg/dl) & $179.3 \pm 36.8$ & $180.9 \pm 38.2$ & 0.83 \\
LDL cholesterol (mg/dl) & $116.5 \pm 35.6$ & $128.7 \pm 33$ & 0.10 \\
HDL cholesterol (mg/dl) & $43.9 \pm 17$ & $50.9 \pm 22.4$ & 0.10 \\
TGL $(\mathrm{mg} / \mathrm{dl})^{\mathrm{a}}$ & $143 \pm 36$ & $150 \pm 45$ & 0.37 \\
Blood glucose $(\mathrm{mg} / \mathrm{dl})^{\mathrm{a}}$ & $135.1 \pm 72.8$ & $116.7 \pm 49.6$ & 0.09 \\
\hline
\end{tabular}

BMI: Body Mass Index; LDL: Low Density Lipoprotein; HDL: High Density Lipoprotein; ${ }^{a}$ Mean \pm standard deviation.

In Table 3, the results according to patient's outcome, adherence to MedDietScore and laboratory findings at 6 months following PCI are presented. As for laboratory findings, compared with control group, intervention group had lower LDL and blood glucose concentration at 6 months following PCI and the same MedDietScore.

A repeated measures ANOVA analysis was applied so as to compare TCHOL, HDLC, LDLC, TGL and blood glucose between 3 time points; during patient's admission to hospital, 3 months and 6 months after hospital discharge. All comparisons were applied to the total studied sample. In Table 4, results 
Table 3. Results according to patient's outcome, adherence to MedDietScore and laboratory findings at 6 months following percutaneous coronary intervention.

\begin{tabular}{cccc}
\hline & $\begin{array}{c}\text { Intervention group } \\
(\mathbf{n}=93)\end{array}$ & $\begin{array}{c}\text { Control Group } \\
(\mathbf{n}=137)\end{array}$ & p-value \\
\hline Hospital resubmission, (Yes, n (\%)) & 0 & $5(6.4)$ & \\
6-months fatal outcome, (Yes, n (\%)) & 0 & $1(1.2)$ & \\
MedDietScore (range 0 - 55) & $33.1 \pm 3.6$ & $33.1 \pm 2.7$ & 0.99 \\
Total cholesterol (mg/dl) & $174.2 \pm 47.1$ & $173.2 \pm 42.2$ & 0.94 \\
LDL cholesterol (mg/dl) & $108.7 \pm 37$ & $122.6 \pm 32.9$ & 0.25 \\
HDL cholesterol (mg/dl) & $47.9 \pm 22$ & $55.2 \pm 30.5$ & 0.41 \\
TGL (mg/dl) & $126 \pm 24$ & $144 \pm 47$ & 0.11 \\
Blood glucose (mg/dl) & $95.3 \pm 28.9$ & $100.3 \pm 19.6$ & 0.43 \\
\hline
\end{tabular}

LDL: Low Density Lipoprotein; HDL: High Density Lipoprotein; ${ }^{a}$ Mean \pm standard deviation.

Table 4. Results according to patients' adherence to MedDietScore and demographic characteristics at 3 and 6 months follow up.

\begin{tabular}{|c|c|c|c|}
\hline \multicolumn{2}{|c|}{ Variables } & \multirow{2}{*}{$\begin{array}{c}\text { Mean } \pm \text { Standard } \\
\text { Deviation }\end{array}$} & \multirow{3}{*}{$\begin{array}{c}p \text {-value } \\
0.02\end{array}$} \\
\hline MedDietScore 3 months & Men & & \\
\hline after PCI & Women & $28.7 \pm 4.0$ & \\
\hline \multirow{3}{*}{$\begin{array}{c}\text { MedDietScore } 6 \text { months } \\
\text { after PCI }\end{array}$} & Men & $33.1 \pm 3.0$ & \multirow{2}{*}{0.57} \\
\hline & Women & $33.7 \pm 2.5$ & \\
\hline & Married & $30.8 \pm 4.5$ & \multirow{4}{*}{$<0.01$} \\
\hline \multirow{3}{*}{$\begin{array}{c}\text { MedDietScore } 3 \text { months } \\
\text { after PCI }\end{array}$} & Unmarried & $28.9 \pm 3.9$ & \\
\hline & Divorced & $29.4 \pm 4.1$ & \\
\hline & Widowed & $27.1 \pm 5.3$ & \\
\hline \multirow{4}{*}{$\begin{array}{c}\text { MedDietScore } 6 \text { months } \\
\text { after PCI }\end{array}$} & Married & $34.6 \pm 2.5$ & \multirow{4}{*}{$<0.01$} \\
\hline & Unmarried & $33.5 \pm 2.9$ & \\
\hline & Divorced & $32.6 \pm 3.0$ & \\
\hline & Widowed & $30.3 \pm 2.5$ & \\
\hline \multirow{4}{*}{$\begin{array}{c}\text { MedDietScore } 3 \text { months } \\
\text { after PCI }\end{array}$} & Bad $(<9000$ euro $)$ & $28.9 \pm 5.2$ & \multirow{4}{*}{0.19} \\
\hline & Moderate (9001 - 18,000 euro) & $30.6 \pm 4.8$ & \\
\hline & Good $(18,001-28,000$ euro $)$ & $30.3 \pm 4.4$ & \\
\hline & Very good (>28,000 euro) & $29.0 \pm 4.8$ & \\
\hline \multirow{4}{*}{$\begin{array}{c}\text { MedDietScore } 6 \text { months } \\
\text { after PCI }\end{array}$} & Bad $(<9000$ euro $)$ & $32.3 \pm 3.7$ & \multirow{4}{*}{0.12} \\
\hline & Moderate (9001 - 18,000 euro) & $33.6 \pm 2.8$ & \\
\hline & Good (18,001-28,000 euro) & $34.3 \pm 2.7$ & \\
\hline & Very good ( $>28,000$ euro) & $32.5 \pm 3.3$ & \\
\hline
\end{tabular}

according to patients' demographic characteristics in relation to MedDietScore, at 3 and 6 months follow up, are presented. 


\subsection{Cholesterol}

A repeated measures ANOVA with a Greenhouse-Geisser correction determined that mean TCHOL concentration differed statistically significantly between the 3 time points $(\mathrm{F}(1.535,49.105)=7.183, p=0.004)$. Post hoc tests using the Bonferroni correction revealed that mean TCHOL concentration while patients in both groups admitted to hospital differed statistically significantly between the time points of 3 and 6 months $(209 \pm 67 \mathrm{mg} / \mathrm{dl}$ vs $174 \pm 34 \mathrm{mg} / \mathrm{dl}$ vs $176 \pm 36$ $\mathrm{mg} / \mathrm{dl}), p=0.005$ and $p=0.042$ respectively. However, there was no statistical significant difference between 3 months and 6 months measurements, $(\mathrm{p}=$ 1.000).

\subsection{HDL}

A repeated measures ANOVA with a Greenhouse-Geisser correction determined that mean HDLC concentration did not differ statistically significantly between the 3 time points $(\mathrm{F}(1.848,51,756)=0.513, p=0.587)$. Post hoc tests using the Bonferroni correction revealed that mean HDLC concentration while patients in both groups admitted to hospital did not differ statistically significantly between the time points of 3 and 6 months $(47 \pm 26 \mathrm{mg} / \mathrm{dl}$ vs $53 \pm 19 \mathrm{mg} / \mathrm{dl}$ vs $52 \pm 28$ $\mathrm{mg} / \mathrm{dl}), p=0,861$ and $p=1.000$ respectively. However, there was no significant statistical difference between 3 months and 6 months measurements, $(p=$ $1.000)$.

\subsection{LDL}

A repeated measures ANOVA with a Greenhouse-Geisser correction determined that mean LDL concentration did not differ statistically significantly between the 3 time points $(\mathrm{F}(1.702,47.664)=2.174, p=0.132)$. Post hoc tests using the Bonferroni correction revealed that mean LDL concentration while patients in both groups admitted to hospital did not differ statistically significantly between the time points of 3 and 6 months $(137 \pm 46 \mathrm{mg} / \mathrm{dl}$ vs $123 \pm 36 \mathrm{mg} / \mathrm{dl}$ vs $120 \pm 28$ $\mathrm{mg} / \mathrm{dl}), p=0.299$ and $p=0.339$ respectively. However, there was no significant statistical difference between 3 months and 6 months measurements, $(p=$ $1.000)$.

\subsection{TGL}

A repeated measures ANOVA with a Greenhouse-Geisser correction determined that mean TGL concentration did not differ statistically significantly between the 3 time points $(\mathrm{F}(1.394,37.645)=9.102, p=0.002)$. Post hoc tests using the Bonferroni correction revealed that mean TGL concentration while patients in both groups admitted to hospital differ statistically significantly between the time points of 3 and 6 months $(167 \pm 58 \mathrm{mg} / \mathrm{dl}$ vs $142 \pm 33 \mathrm{mg} / \mathrm{dl}$ vs $136 \pm 21$ $\mathrm{mg} / \mathrm{dl}), p=0.009$ and $p=0.010$ respectively. However, there was no significant statistical difference between 3 months and 6 months measurements, $(p=$ 0.798). 


\subsection{Glucose}

A repeated measures ANOVA with a Greenhouse-Geisser correction determined that mean blood glucose differ statistically significantly between the 3 time points $(\mathrm{F}(1.715,94.304)=7.522, p=0.002)$. Post hoc tests using the Bonferroni correction revealed that mean glucose concentration while patients in both groups admitted to hospital differ statistically significantly between the time points of 3 and 6 months $(108 \pm 40 \mathrm{mg} / \mathrm{dl}$ vs $95 \pm 21 \mathrm{mg} / \mathrm{dl}$ vs $95 \pm 23 \mathrm{mg} / \mathrm{dl}), p$ $=0.009$ and $p=0.012$ respectively. However, there was no significant statistical difference between 3 months and 6 months measurements, $(p=1.000)$.

\section{Discussion}

According to the results of the present study, nursing intervention as regards the dietary habits after coronary artery angioplasty had a beneficial effect, as patients made an effort and achieved to control their blood glucose levels, 3 and 6 months after PCI. Although the intervention was also focused on lowering their lipid levels, there was no significant difference on their blood test results 3 or 6 months after their hospital discharge. A possible explanation of this finding is the fact that patients are not able to adjust with the new changes in their dietary habits and probably seek for more time to comply with the new lifestyle changes. Thus, it could be hypothesized that by providing more time to patients so as to follow the dietary recommendations, a significant difference could be achieved on controlling patients' lipid levels.

In contrast to the present study, Anderson et al. [16] evaluated the effect of a behaviorally oriented secondary prevention program on lifestyle changes of patients treated with PCI. The follow up time was at 12, 24, 36 and 60 months after their hospital discharge and it was observed significantly lower rates of recurrent events and of cardiovascular mortality in the intervention group. Another approach to this finding is the differences of patients' socioeconomic status.

According to the results of the present study, patients' marital status seems to affect their adherence to MD pattern but it was not observed the same with their financial status. In contrary to our findings, Bonaccio et al. [17] in their crosssectional study tried to examine associations of socioeconomic status with adherence to a MD pattern. According to their results, household higher income was significantly associated with greater adherence to a MD pattern. They also underlined that education level is independently associated with a great adherence to MD eating patterns.

Similarly, Erkkilä et al. [18] in their study supported that patients' socioeconomic status and mainly their educational level, may influence their dietary habits after a cardiac event. Panagiotakos et al. [19] also in their work investigated whether dietary habits are associated with socio-economic status. They concluded that groups with low socio-economic status showed less adherence to the Mediterranean diet compared with high socio-economic status groups; thus, in the first groups are observed higher CVD risk factors profile.

According to literature, patients who underwent to coronary angioplasty need 
to follow a specific eating pattern and adjust their eating habits in order to achieve better health outcomes and direct against heart disease progression [20] [21].

While literature supports that women are more interested in following a healthier dietary pattern than men [22], in our study a statistical significant difference between the gender and the adherence in Med Diet pattern was found, with men showing better scoring values than women. Approximately, our results revealed that married patients were more likely to comply with Med Diet than those who were unmarried, divorced or widowed. This may be explained by the traditional role of men and women in households, since married subjects are more responsible for preparing the meals and take the dietary habits into serious consideration than those who are unmarried, divorced or widowed.

Leblanc et al. [23] in their study tried to determine gender differences in long-term effects of a 12-week nutritional intervention program promoting the adoption of the MD pattern in men and women presenting cardiovascular risk factors. Their results demonstrated that men achieved more beneficial changes in long-term dietary intakes and showed greater improvement to their metabolic profile than women.

The association between healthy dietary habits with the presence of less acute coronary syndromes and better short-term prognosis became the subject of study for Panagiotakos et al. [6]. According to their findings, patients who followed healthy dietary habits based on Mediterranean pattern were less likely to have severe cardiac events and lower risk of death or rehospitalization 30 days after the event.

\section{Limitation of the Study}

Our study has some limitations. The main limitation of the present study was the small sample size. In addition to that, culture and ethnicity are factors which may both play an important role in patients' adherence with dietary advice but were not taken into consideration in the present study. Also, it may have been response bias due to misreporting or underreporting by patients who knew that they participated in an intervention trial and tried harder to perform better.

\section{Conclusion}

The findings of the present study suggest that a nurse-led program regarding dietary habits modifications with a long term follow-up is of high importance for patients undergoing percutaneous coronary intervention.

\section{Conflicts of Interest}

The authors declare that there is no conflict of interest regarding the publication of this article.

\section{References}

[1] World Health Organization (2014) Global Status Report on Noncommunicable 
Diseases 2010. Geneva.

http://www.who.int/nmh/publications/ncd_report_full_en.pdf

[2] Tran, D.M. and Zimmerman, L.M. (2015) Cardiovascular Risk Factors in Young Adults: A Literature Review. Journal of Cardiovascular Nursing, 4, 298-310. https://doi.org/10.1097/JCN.0000000000000150

[3] Berra, K. (2010) Challenges of Changing Lifestyle to Reduce Risk for Cardiovascular Disease. Journal of Cardiovascular Nursing, 3, 223-227. https://doi.org/10.1097/JCN.0b013e3181cec7e4

[4] Pahkala, K., Niinikoski, H. and Raitakari, O. (2014) Prevention of Cardiovascular Diseases-The Most Recent Recommendations from the United States. Duodecim, 8, 778-784.

[5] Bautista, M.C. and Engler, M.M. (2005) The Mediterranean Diet: Is It Cardioprotective? Progress in Cardiovascular Nursing, 2, 70-76. https://doi.org/10.1111/j.0889-7204.2005.04558.x

[6] Panagiotakos, D.B., Pitsavos, C., Polychronopoulos, E., Chrysohoou, C., Zampelas, A. and Trichopoulou, A. (2004) Can a Mediterranean Diet Moderate the Development and Clinical Progression of Coronary Heart Disease? A Systematic Review. Medical Science Monitor, 8, 193-198.

[7] Panagiotakos, D.B., Pitsavos, C., Chrysohoou, C., Stefanadis, C. and Toutouzas, P. (2002) Primary Prevention of Acute Coronary Events through the Adoption of a Mediterranean-Style Diet. Eastern Mediterranean Health Journal, 4-5, 593-602.

[8] Hall, S.L. and Lorenc, T. (2010) Secondary Prevention of Coronary Artery Disease. American Family Physician, 3, 289-296.

[9] Hu, F.B. (2009) Diet and Lifestyle Influences on Risk of Coronary Heart Disease. Current Atherosclerosis Reports, 4, 257-263. https://doi.org/10.1007/s11883-009-0040-8

[10] Hata, Y. and Nakajima, K. (2000) Life-Style and Serum Lipids and Lipoproteins. Journal of Atherosclerosis and Thrombosis, 4, 177-197. https://doi.org/10.5551/jat1994.7.177

[11] Farrell, T.C. and Keeping-Burke, L. (2014) The Primary Prevention of Cardiovascular Disease: Nurse Practitioners Using Behaviour Modification Strategies. Canadian Journal of Cardiovascular Nursing, 1, 8-15.

[12] Haskell, W.L. (2003) Cardiovascular Disease Prevention and Lifestyle Interventions: Effectiveness and Efficacy. Journal of Cardiovascular Nursing, 4, 245-255. https://doi.org/10.1097/00005082-200309000-00003

[13] Cao, Y., Davidson, P.M. and DiGiacomo, M. (2009) Cardiovascular Disease in China: An Urgent Need to Enhance the Nursing Role to Improve Health Outcomes. Journal of Clinical Nursing, 5, 687-693.

[14] Panagiotakos, D.B., Pitsavos, C., Arvaniti, F. and Stefanadis, C. (2007) Adherence to the Mediterranean Food Pattern Predicts the Prevalence of Hypertension, Hypercholesterolemia, Diabetes and Obesity, among Healthy Adults; the Accuracy of the MedDietScore. Preventive Medicine, 4, 335-340. https://doi.org/10.1016/j.ypmed.2006.12.009

[15] Rassaf, T., Steiner, S. and Kelm, M. (2013) Postoperative Care and Follow-Up after Coronary Stenting. Deutsches Ärzteblatt International, 5, 72-81. https://doi.org/10.3238/arztebl.2013.0072

[16] Anderson, L. and Taylor, R.S. (2014) Cardiac Rehabilitation for People with Heart Disease: An Overview of Cochrane Systematic Reviews. The Cochrane Database of 
Systematic Reviews, 12, CD011273. https://doi.org/10.1002/14651858.CD011273

[17] Bonaccio, M., Bonanni, A.E., Di Castelnuovo, A., De Lucia, F., Donati, M.B., de Gaetano, G. and Iacoviello, L. (2012) Moli-Sani Project Investigators. Low Income Is Associated with Poor Adherence to a Mediterranean Diet and a Higher Prevalence of Obesity: Cross-Sectional Results from the Moli-Sani Study. BMJ Open, 6, e001685.

[18] Erkkilä, A.T., Sarkkinen, E.S., Lehto, S., Pyörälä, K. and Uusitupa, M.I. (1999) Diet in Relation to Socioeconomic Status in Patients with Coronary Heart Disease. $E u$ ropean Journal of Clinical Nutrition, 8, 662-668. https://doi.org/10.1038/sj.ejcn.1600829

[19] Panagiotakos, D.B., Pitsavos, C., Chrysohoou, C., Vlismas, K., Skoumas, Y., Palliou, K. and Stefanadis, C. (2008) Dietary Habits Mediate the Relationship between Socio-Economic Status and CVD Factors among Healthy Adults: The ATTICA Study. Public Health Nutrition, 12, 1342-1349. https://doi.org/10.1017/S1368980008002978

[20] Proença Vieira, L., Nobre, M.R. and Gonçalves de Queiroz, G. (2012) Eating Behavior and Nutritional Status in Patients Who Underwent Coronary Angioplasty. $\mathrm{Nu}$ tricion Hospitalaria, 1, 281-287.

[21] Booth, J.N., Levitan, E.B., Brown, T.M., Farkouh, M.E., Safford, M.M. and Muntner, P. (2014) Effect of Sustaining Lifestyle Modifications (Nonsmoking, Weight Reduction, Physical Activity, and Mediterranean Diet) after Healing of Myocardial Infarction, Percutaneous Intervention, or Coronary Bypass (from the REasons for Geographic and Racial Differences in Stroke Study). American Journal of Cardiology, 12, 1933-1940. https://doi.org/10.1016/j.amjcard.2014.03.033

[22] López-Azpiazu, I., Martinez-González, M.A., León-Mateos, A., Kearney, J., Gibney, M. and Martínez, J.A. (2000) Stages of Dietary Change and Nutrition Attitudes in the Spanish Population. Public Health, 3, 183-189. https://doi.org/10.1016/S0033-3506(00)00332-2

[23] Leblanc, V., Bégin, C., Hudon, A.M., Royer, M.M., Corneau, L., Dodin, S. and Lemieux, S. (2014) Gender Differences in the Long-Term Effects of a Nutritional Intervention Program Promoting the Mediterranean Diet: Changes in Dietary Intakes, Eating Behaviors, Anthropometric and Metabolic Variables. Nutrition Journal, 13, 107. https://doi.org/10.1186/1475-2891-13-107 